\title{
Verfahrensrechte bei medizinischen Begutachtungen
}

\author{
Marco Weiss \\ Dr. iur., Auditor, SVA St Gallen. Der Autor gibt seine persönliche Meinung wieder und bindet seinen Arbeitgeber nicht.
}

Jedes Jahr verfassen Ärzte mehrere tausend Gutachten für die Unfall-, Invaliden- oder Krankenversicherer. Ohne medizinische Expertise könnten die Ansprüche im gesamten Sozialversicherungsrecht heute nicht mehr beurteilt werden. In der jüngsten juristischen Lehre und Rechtsprechung werden vermehrt die Verfahrensrechte der zu begutachtenden Person in den Vordergrund gestellt, weshalb diese von den medizinischen Gutachtern unbedingt beachtet werden müssen, um ein rechtskonformes medizinisches Gutachten erstellen zu können [1]. Dieser Beitrag gibt eine Übersicht über jene Verfahrensrechte der zu begutachtenden Person, die der Arzt während der medizinischen Exploration auf jeden Fall berücksichtigen muss bzw. einräumen kann.

\section{Anspruch auf einen unbefangenen und unabhängigen Gutachter}

Gemäss Art. 29 Abs. 1 BV hat jede Person in Verfahren vor Gerichts- und Verwaltungsinstanzen Anspruch auf gleiche und gerechte Behandlung sowie auf Beurteilung innert angemessener Frist. Aus dieser verfassungsrechtlich verankerten Fairnessgarantie leitet sich ab, dass eine zu begutachtende Person im Sozialversicherungsrecht einen Anspruch auf eine unabhängige und unbefangene Entscheidbehörde hat, sodass daraus folgt, dass ein Arzt als Sachverständiger innerhalb des Sozialversicherungsverfahrens aus persönlichen oder anderen Befangenheitsgründen in den Ausstand zu treten

\section{Résumé}

Chaque année, les médecins rédigent des milliers d'expertises pour les assurances-accidents, invalidité et maladie. Sans expertises médicales, il serait aujourd'hui impossible d'évaluer les revendications dans tout le domaine du droit des assurances sociales. La doctrine juridique et la jurisprudence récentes accordent davantage d'importance aux droits de procédure de la personne à examiner, c'est pourquoi les experts médicaux doivent absolument en tenir compte afin de pouvoir rédiger une expertise médicale conforme au droit [1]. Cet article propose une vue d'ensemble desdits droits de procédure de la personne soumise à l'expertise que le médecin doit absolument prendre en considération ou qu'il peut accorder lors de l'exploration médicale. hat und somit an der medizinischen Beweiserhebung nicht mehr mitwirken darf [2]. Dieser verfassungsrechtliche Anspruch ist für das gesamte Sozialversicherungsverfahren in Art. 36 Abs. 1 ATSG bundesgesetzlich konkretisiert worden. Nach dieser Gesetzesnorm haben Personen, die Entscheidungen über Rechte und Pflichten zu treffen oder vorzubereiten haben, in Ausstand zu treten, wenn sie in der Sache ein persönliches Interesse haben oder aus anderen Gründen in der Sache befangen sein könnten [3]. Zu den letztgenannten «anderen Gründen» im Sinne von Art. 36 Abs. 1 ATSG gehören insbesondere persönliche Beziehungen in Form von Feind- und Freundschaft, wirtschaftliche Interessen oder gegenüber der zu begutachtenden Person gemachte Äusserungen herabwürdigender Art [4].

Bei externen Gutachterstellen wie den MEDAS gilt zudem die Besonderheit, dass die zu begutachtende Person den Gutachter nach Art. 44 ATSG aufgrund sogenannter triftiger Gründe ablehnen kann. Dies erlaubt einen medizinischen Experten aufgrund mangelnder medizinischer Fachausbildung (Bsp.: Fehlender Spezialarzttitel oder fehlende Fachausbildung als Neuropsychologe) abzulehnen [5].

Der Arzt hat sich vor der medizinischen Begutachtung also immer zu fragen, ob einer der beispielhaft genannten Ausstandsgründe bejaht werden kann oder nicht. Diese Aspekte haben selbstverständlich auch während der medizinischen Begutachtung und bei Er- 
stellung des Gutachtens zu gelten. Sofern diese Standards nicht mehr gewährleistet sein sollten, hat der Gutachter im Idealfall von sich selbst aus in den Ausstand zu treten. Andernfalls haben die Gerichte zu entscheiden, ob der Arzt als Gutachter unabhängig agiert hat und das medizinische Gutachten als Beweismittel verwertbar ist oder nicht.

\section{Beizug eines Dolmetschers}

Oftmals hat zu einer medizinischen Begutachtung zur Klärung von Leistungsansprüchen im Unfall-, Invalidenversicherungs- oder Krankenversicherungsrecht ein Dolmetscher hinzugezogen zu werden, weil die zu begutachtende Person entweder gar nicht bzw. nur bedingt mit einer der schweizerischen Amtssprachen vertraut ist. Grundsätzlich besteht keinerlei Anspruch auf Durchführung einer medizinischen Exploration in der Muttersprache der zu begutachtenden Person [6]. Die Rechtspraxis lässt es aber grundsätzlich

\section{Angehörige sollten vom Arzt nicht als Dolmetscher herangezogen werden.}

in der Kompetenz des medizinischen Experten, inwiefern ein Dolmetscher zur medizinischen Begutachtung geeignet erscheint oder nicht. Dies bedeutet, dass der Arzt zu entscheiden hat, ob der Beizug eines Dolmetschers angebracht ist oder nicht [7]. Angehörige sollten vom Arzt nicht als Dolmetscher herangezogen werden, weil das Bundesgericht davon ausgeht, dass diese aufgrund mangelnder Distanz zum Exploranden und des Zwanges zu "familienrollenkonformem» Verhaltens als ungeeignet erscheinen. [8].

Eine bedeutende Ausnahme von dieser Praxis lässt die Rechtsprechung bei psychiatrischen Untersuchungen gelten. Aus Sicht der Rechtsprechung ist bei psychiatrischen Untersuchungen ein Dolmetscher beizuziehen, sofern sprachliche Schwierigkeiten bestehen und das Gespräch nicht in der Muttersprache des Exploranden bzw. der Explorandin geführt werden kann [9]. Die Gerichte anerkennen hier, dass bei psychiatrischen Untersuchungsgesprächen eine bestmögliche Verständigung notwendig ist. Dies umfasst auch die Spontaneität, den Tonfall und die nonverbalen Äusserungen in Form von Mimik und Gestik, mit denen sich der Explorand bei einer psychiatrischen Untersuchung ausdrückt [10]. Eine weitere Ausnahme besteht bei Begutachtungen im Zusammenhang mit externen Gutachterstellen wie den MEDAS. Hier besteht ein Anspruch für die zu begutachtende Person, dass die Exploration in einer ihr verständlichen schweizerischen Amtssprache stattfindet [11].

Dem Arzt als medizinischem Gutachter obliegt somit eine grosse Verantwortung, da es zu seinen Kompetenzen gehört, ob zu einer Exploration der Beizug eines Dolmetschers notwendig erscheint oder nicht.

\section{Beizug von Angehörigen und anderen Personen}

Eine ähnlich weitreichende Kompetenz besteht für den Arzt bei der Frage, ob es statthaft ist, dass Angehörige

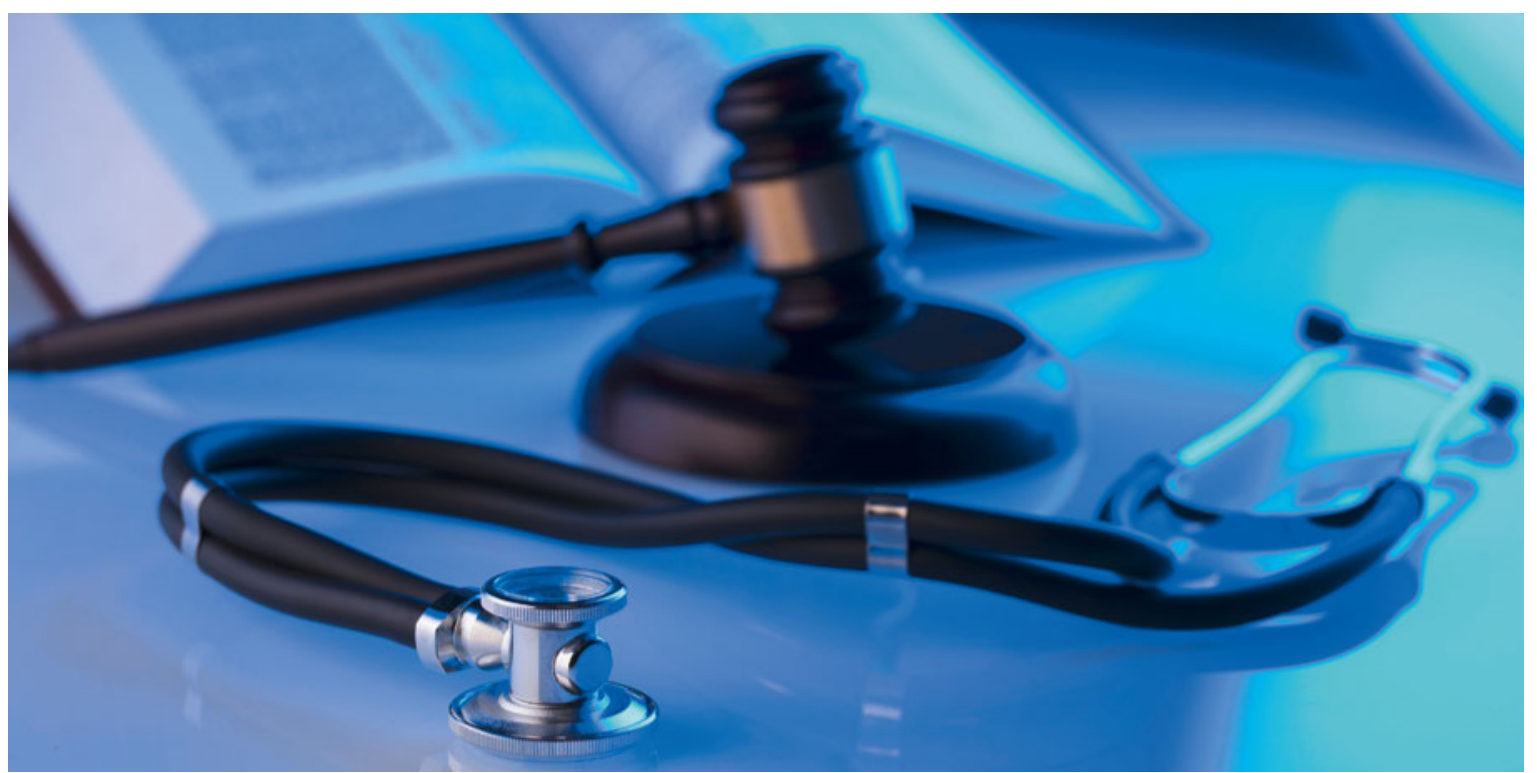

Wenn Ärzte Gutachten für die Unfall-, Invaliden- oder Krankenversicherer erstellen, müssen sie die Verfahrensrechte der zu begutachtenden Person unbedingt beachten. 
der zu begutachtenden Person oder Personen aus ihrem Vertrauenskreis (Bsp.: Rechtsvertreter oder -berater) an der Untersuchung teilnehmen dürfen oder nicht.

\section{Es obliegt der letztendlichen Kompetenz des Arztes, ob Angehörige zur Exploration zugelas- sen werden oder nicht.}

Bezüglich der Frage, ob Angehörige die zu begutachtende Person zu der Exploration begleiten dürfen, hält die Rechtsprechung fest, dass hierzu grundsätzlich kein Anspruch besteht. Allerdings obliegt es der letztendlichen Kompetenz des Arztes, ob Angehörige zur Exploration zugelassen werden oder nicht. Entscheidend ist hier also, ob der Arzt dies als notwendig erachtet oder nicht [12]. Hinsichtlich der Frage, ob sich die zu begutachtende Person während der Begutachtung verbeiständen lassen kann, vertritt die Rechtsprechung eine härtere Linie, weil hierzu grundsätzlich kein Anspruch besteht. Aus Sicht der Rechtsprechung soll der Arzt deshalb keinen Rechtsvertreter zur medizinischen Begutachtung hinzuziehen, weil so die Interaktion zwischen Experten und Explorand gestört werden kann. Anders zu entscheiden würde nach dem Bundesgericht bedeuten, dass die Rahmenbedingungen für eine ordnungsgemässe und wissenschaftliche Begutachtung nicht mehr gewährleistet sein können [13].

\section{Fazit}

Korrespondenz: SVA St. Gallen

Brauerstrasse 54

CH-9016 St. Gallen

Vor und während der medizinischen Exploration hat der Arzt als medizinischer Sachverständiger im Sozialversicherungsrecht einige Verfahrensrechte der zu begutachtenden Person zur Kenntnis zu nehmen. Betreffend des Anspruchs des Exploranden auf eine unabhängige und unbefangene Begutachtung hat sich der Arzt selbst und frühzeitig zu fragen, ob er diesen Anspruch gewährleisten kann oder nicht. In Zweifelsfällen sollte er auf jeden Fall von sich aus in den Ausstand treten. Hinsichtlich des Anspruchs des Exploranden auf Beizug eines Dolmetschers oder Angehörigen zur Begutachtung hat sich der Arzt bewusst zu werden, dass ihm hier eine hohe Entscheidkompetenz zukommt.

\section{Literatur}

1 Grundlegend BGE 137 V 210; Massimo Aliotta. Begutachtungen im Bundessozialversicherungsrecht. Gehörs- und Partizipationsrechte der versicherten Personen bei Begutachtungen im nichtstreitigen Verwaltungsverfahren gemäss ATSG. Zürich/Basel/ Genf: Schulthess; 2017; Marco Weiss. Mitwirkungsrechte vor der Einholung medizinischer Gutachten in der Invalidenversicherung. Problematiken und Regelungsmöglichkeiten. Bern: Editions Weblaw; 2018. Vgl. auch BGE 134 V 231 E. 5.1; 125 V 351 E. 3a zu den Anforderungen der Rechtsprechung an eine medizinische Expertise.

2 Art. 29 Abs. 1 BV i.V.m. Art. 36 Abs. 1 ATSG.

3 Art. 36 Abs. 1 ATSG.

4 Insbesondere BGE 137 V 210 E. 2.4 ff. und 3.4.1 ff.; 120 V 157 E. 3b; Urteil des Bundesgerichts 9C 893/2009 vom 22. Dezember 2009 E. 1.2.2.

5 BGE 132 V 93 E. 6.4; Urteil des Versicherungsgerichts des Kantons St. Gallen IV 2016/432 vom 15. Februar 2017 E. 3.

6 Urteil des Bundesgerichts 8C_578/2014 vom 17. Oktober 2014 E. 4.2.4.

7 Urteil des Eidgenössischen Versicherungsgerichts I 28/06 vom 28. April 2006 E. 3.1

8 BGE 140 V 260 E. 3.2.3 f

9 BGE 140 V 260 E. 3.2.1.

10 Urteil des Bundesgerichts 8C 578/2014 vom 17. Oktober 2014 E. 4.2.5.

11 BGE 128 V 34 E. $2 a ; 127$ V 219 E. $2 \mathrm{~b} / \mathrm{bb}$.

12 BGE 140 V 260 E. 3.2.3.

13 BGE 137 V 210 E. 3.1.3.3; 132 V 443 E. 3.5.
Bildnachweis

(c) Franky44 | Dreamstime.com 